\title{
Introduction to "Fast matrix computations for functional additive models" by S. Barthelmé
}

\author{
Håvard Rue
}

Published online: 1 November 2014

(C) Springer Science+Business Media New York 2014

I am very enthusiastic about the very innovative paper "Fast matrix computations for functional additive models" by S. Barthelmé, which I find both to be both methodologically and practically very useful. The characterization of the new rQK-class of symmetric positive definite matrices (with inspiration from previous work of Heersink and Furrer (2011)), defines a new class of matrices which can be computed with much less computational costs compared to the general case. Further, the rQK-class is closed under multiplication and inversion. This adds new tools to the computational statistician's toolbox, in addition to the well known computational friendly block-diagoal, Toeplitz, Toeplitz circulant and sparse matrices. Barthelmé demonstrates, con- vincingly, how to leverage this new rQK-class to obtain great computational savings doing inference in some Latent Gaussian models for functional data analysis.

\section{References}

Heersink, D.K., Furrer, R.: On Moore-Penrose inverses of quasiKronecker structured matrices. Linear Algebra Appl. 436, 561570 (2011)
H. Rue $(\varangle)$

Department of Mathematical Sciences, NTNU, Trondheim, Norway

e-mail: Havard.Rue@ntnu.no 